\section{A new model to explain the redundant-signals effect ${ }^{1}$}

\author{
W. SCHWARZ \\ Philipps Universität \\ Marburg, Federal Republic of Germany
}

The typical reaction time (RT) experiment on the redundant-signals effect (RSE) (Gielen, Schmidt, \& van den Heuvel, 1983; Miller, 1982) entails a comparison of three conditions: The redundant-signal trials, in which two stimuli are presented, are compared with the singlesignal trials, in which one of the two stimuli is presented alone. In most cases, an auditory and a visual stimulus are employed. The RSE, or at least an instance of it, is that the RT in redundant-signal trials is shorter than the $\mathrm{RT}$ in single-signal trials in a stochastic sense.

In an influential paper, Raab (1962) considered a purely statistical explanation of this effect: In the redundant-signal trials, the subject responds simply to the signal that is transmitted first to a central decision stage onto which both stimulated peripheral channels converge. Denoting the latencies of the auditory and visual channels from the presentation of the appropriate stimulus to the central response as $T_{A}, T_{V}$, respectively, his model states that

$$
T_{R}=\min \left(T_{A}, T_{V}\right),
$$

where $T_{R}$ is the latency to the central decision to execute the response in the redundant-signal trials.

The common finding (Diederich \& Colonius, 1987; Miller, 1982, 1986) is that although this sort of facilitation may well be present, it is alone insufficient to explain the amount of the RT reduction in the redundantsignal trials as inferred from the single-signal trials. This holds true even if the laterlcies of the two channels are allowed to covary negatively in order to maximize the RSE (Colonius, 1986; Ulrich \& Giray, 1986).

In contrast to the thoroughness with which Raab's "nullmodel" (Equation 1) has been formulated and tested, there is as yet no satisfactory model to explain the RSE. Miller (1986, p. 338) has described an "exponential coactivation model" that is also incapable of explaining the total effect. Colonius (1988) has shown that the same holds true if one drops the exponential assumption.

Hitherto, the only alternative has been the accumulation model of Grice, Canham, and Boroughs (1984), which, however, makes very strong deterministic assumptions concerning the accumulation of sensory information. In the light of newer evidence (Luce, 1986, chaps. 8-9; Ratcliff, 1988), these deterministic assumptions are prob-

Correspondence may be addressed to W. Schwarz, Philipps Universität, Fachbereich Psychologie, Gutenbergstrasse 18, D-3550 Marburg, Federal Republic of Germany. ably an oversimplification. Furthermore, Miller (1986) has shown that the model of Grice et al. (1984) cannot explain his redundant-signal RTs if the two signals are not presented simultaneously. The aims of this note are both to present a stochastic model that can fully explain the RSE and to illustrate its scope using the stimulus onset asynchrony (SOA) data of Miller (1986).

\section{The Superposition Model}

Consider, first, single-signal trials, and assume that the presentation of the stimulus induces a neural renewal counting process $\left\{N_{A}(t), t \geq 0\right\}$ (e.g., see Green \& Luce, 1974). Let $T_{i}, i \geq 1$, denote the waiting time for the $i$ th count. In an ordinary renewal process (Smith, 1988), the intercount intervals $Z_{1} \equiv T_{1}$ and $Z_{i} \equiv T_{i}-T_{i-1}, i>1$, are independently and identically distributed. Assume that the central decision to execute the response is reached as soon as $c$ counts have been registered.

The main assumption for the redundant-signal trials is that the two renewal processes are simply superposed-that is, we define a stochastic process $N_{R}(t) \equiv N_{A}(t)+N_{V}(t)$ and consider again the waiting time $T_{c}$ to the $c$ th renewal count. It is intuitively obvious that the $c$ counts will be collected earlier in the superposed process $N_{R}(t)$, but for serious work we need the exact relation between the individual channel latencies and the latency on redundantsignal trials.

Denote as $P_{k}\left(T_{c} \leq t\right), k=A, V, R$, the distribution function of $T_{c}$ under the auditory single-signal, visual singlesignal, and redundant-signal trials, respectively. Our aim, then, is to find $P_{R}\left(T_{c} \leq t\right)$ in terms of the $P_{A}\left(T_{c} \leq t\right)$, $P_{V}\left(T_{c} \leq t\right)$. In Appendix $\mathrm{A}$, it is shown that

$$
\begin{array}{r}
1-P_{R}\left(T_{c} \leq t\right)=\sum_{n=0}^{c-1} \sum_{i=0}^{n}\left[P_{A}\left(T_{i} \leq t\right)-P_{A}\left(T_{i+1} \leq t\right)\right] \\
{\left[P_{V}\left(T_{n-i} \leq t\right)-P_{V}\left(T_{n-i+1} \leq t\right)\right],}
\end{array}
$$

where $P_{k}\left(T_{0} \leq t\right) \equiv P\left[N_{k}(t) \geq 0\right]=1$.

Equation 2 gives (one minus) the distribution function of the waiting time $T_{c}$ for the $c$ th count in the superposed process $N_{R}(t)=N_{A}(t)+N_{V}(t)$, in terms of the waitingtime distributions of the auditory and the visual channel. In general, $N_{R}(t)$ is not again a renewal process with independent and identically distributed intercount intervals. For the special case $c=1$, Equation 2 reduces to Raab's model (Equation 1) with independent channels, as is clear from the construction of the process $N_{R}(t)$.

Of course, the apparent generality of the result (Equation 2) is somewhat misleading. For its general use, we need both sequences of the functions $\left\{P_{A}\left(T_{n} \leq t\right)\right.$, $n \geq 1\},\left\{P_{V}\left(T_{n} \leq t\right), n \geq 1\right\}$. Practically, this amounts to finding a stable or at least divisible distribution for the intercount intervals $Z$ (Feller, 1966, pp. 165ff.). Thus, two obvious choices for the densities $g(z)$ of $Z$ are 


$$
g(z)=\alpha_{k} \exp \left(-\alpha_{k} z\right)
$$

and

$$
g(z)=\left(\frac{\delta_{k}}{2 \pi z^{3}}\right)^{1 / 2} \exp \left[-\delta_{k} \frac{\left(z-\mu_{k}\right)^{2}}{2 z \mu_{k}^{2}}\right] \quad k=A, V .
$$

The first case refers to the homogeneous Poisson process with its exponential intercount intervals (with a rate that depends on which stimulus was presented). For this process, the density $f_{n}(t)$ of the waiting time $T_{n}$ to the $n$th count is well known to be

$$
f_{n}(t)=\frac{\alpha_{k}\left(\alpha_{k} t\right)^{n-1} \exp \left(-\alpha_{k} t\right)}{(n-1) !}
$$

The second case refers to intercount intervals distributed according to Wald's first passage-time distribution-that is, the time to the first crossing of a predescribed level in a Brownian motion. For this process, the density $f_{n}(t)$ of the waiting time $T_{n}$ to the $n$th count is

$$
f_{n}(t)=\left(\frac{n^{2} \delta_{k}}{2 \pi t^{3}}\right)^{1 / 2} \exp \left[-n^{2} \delta_{k} \frac{(t-n \mu)^{2}}{2 t\left(n \mu_{k}\right)^{2}}\right] .
$$

Luce $(1986$, pp. 145, 334) gives applications of this family to RTs.

\section{Prediction of SOA-Dependent RT Data}

The application of the superposition model to SOA data as given by Miller (1986) is straightforward. Assume that the visual stimulus is presented $\tau(\tau \in R)$ msec apart from the auditory stimulus. If $\tau>0$, the visual stimulus is presented $\tau$ msec before the auditory stimulus; if $\tau<0$, the auditory stimulus is presented $-\tau$ msec before the visual stimulus. We consider the process

$$
N_{R, \tau}(t)= \begin{cases}N_{A}(t)+N_{V}(t+\tau) & \text { if } \tau \leq 0 \\ N_{V}(t)+N_{A}(t-\tau) & \text { if } \tau \geq 0,\end{cases}
$$

where, of course, $N_{A}(t)=0, N_{V}(t)=0$ for $t<0$.

The interpretation of Equation 3 is that on redundantsignal trials with SOA $\tau$, each channel starts a renewal counting process upon the presentation of the appropriate stimulus, and that both outputs are superposed to form the relevant overall counting process. As before, we assume that the central decision to respond is reached as soon as the $c$ th count of the process $N_{R, \tau}(t)$ is registered. For $\tau=0$, this construction reduces to the one given in the discussion of the superposition model above.

\section{Application to the Data of Miller (1986)}

To apply the foregoing developments to the SOA-RT data of Miller (1986), let us assume that the individual channels form homogeneous Poisson renewal counting processes. Thus, for an SOA equal to $\tau$ msec, we consider the process $N_{R, \tau}(t)$ and study the distribution $P_{R, \tau}\left(T_{c} \leq t\right)$ of the waiting time $T_{\mathrm{c}}$ to the $c$ th count in $N_{R, \tau}$. In Appendix $\mathrm{B}$, we show that for an SOA $\tau \geq 0$, we have

$$
\begin{aligned}
& 1-\exp \left(-\alpha_{V} t\right) \sum_{i=0}^{c-1} \frac{\left(\alpha_{V} t\right)^{i}}{i !} \quad 0 \leq t \leq \tau \\
& P_{R, \tau}\left(T_{c} \leq t\right)= \\
& 1-\exp \left\{-\left[\alpha_{V} \tau+\left(\alpha_{V}+\alpha_{A}\right)(t-\tau)\right]\right\} \\
& \sum_{i=0}^{c-1} \frac{\left[\alpha_{V} \tau+\left(\alpha_{V}+\alpha_{A}\right)(t-\tau)\right]^{i}}{i !} \quad \tau \leq t
\end{aligned}
$$

where $\alpha_{A}, \alpha_{V}$ denote the intensity parameter of the auditory and the visual channel, respectively. For $\tau<0$, if the auditory stimulus precedes the visual stimulus, we must simply replace $\tau$ by $-\tau(>0)$, and exchange $\alpha_{A}$ and $\alpha_{V}$.

The corresponding expectation $E_{\tau}\left[T_{c}\right]$ for $\tau \geq 0$ is

$$
\frac{c}{\alpha_{V}}-\frac{\alpha_{A}}{\alpha_{V}\left(\alpha_{V}+\alpha_{A}\right)} \exp \left(-\alpha_{V} \tau\right) \sum_{i=0}^{c-1} \frac{\left(\alpha_{V} \tau\right)^{i}}{i !}(c-i),
$$

while for $\tau<0$, remarks similar to those above apply.

Finally, we assume the total RT to be additively composed of $T_{\mathrm{c}}$ and all processes following it. We denote the duration of these latter processes by the random variable $M$. In general, $T_{c}$ and $M$ will not be stochastically independent (e.g., see Ulrich \& Stapf, 1984). However, on the level of the expected RT, we still have

$$
E_{\tau}[\mathrm{RT}]=E_{\tau}\left[T_{c}+M\right]=E_{\tau}\left[T_{c}\right]+E[M] .
$$

This special version of the superposition model was fitted to the SOA-RT data of Miller (1986). For details of this experiment, we simply refer to Miller's original paper. Table 1 contains empirical and predicted mean RT for each SOA.

The fit is quite close and it reproduces all the main features of the data: both asymptotic behaviors, the maximal RSE for $S O A=0$, and the asymmetry of the SOA curve. In addition, it may be noted that in contrast to Raab's race model and its various generalizations, the superposition model can easily cope with substantially larger RSEs than the comparatively small ones in Miller's (1986) study.

Table 1

Fit of Superposition Model to Miller's (1986) Data

\begin{tabular}{rccccc}
\hline & \multicolumn{2}{c}{ Subject B.D. } & & \multicolumn{2}{c}{ Subject K.Y. } \\
\cline { 2 - 3 } \cline { 5 - 6 } SOA & Mean RT & Prediction & & Mean RT & Prediction \\
\hline$-\infty$ & 231 & 231 & & 211 & 215 \\
-167 & 234 & 231 & & 216 & 215 \\
-133 & 230 & 231 & & 217 & 215 \\
-100 & 227 & 230 & & 214 & 215 \\
-67 & 228 & 227 & & 218 & 215 \\
-33 & 221 & 222 & & 215 & 213 \\
0 & 217 & 214 & & 208 & 208 \\
33 & 238 & 238 & & 237 & 232 \\
67 & 263 & 261 & & 249 & 251 \\
100 & 277 & 282 & & 256 & 263 \\
133 & 298 & 299 & & 273 & 271 \\
167 & 316 & 313 & & 278 & 276 \\
$+\infty$ & 348 & 348 & 282 & 283 \\
\hline
\end{tabular}

Note-Parameters: B.D., $M=165, C=3, \alpha_{A}=1 / 22, \alpha_{V}=1 / 61$; K.Y., $M=185, C=2, \alpha_{A}=1 / 15, \alpha_{V}=1 / 49$. 
Although this result is quite encouraging for future work with the superposition model, it is obvious that much stronger tests than the simple fitting of mean RTs are called for. One possibility is to consider predictions for the entire RT distribution; another one is to test the obvious applications of the model to related detection, deadline, temporal order, or go/no-go paradigms.

\section{REFERENCES}

Colonius, H. (1986). Measuring channel dependence in separate activation models. Perception \& Psychophysics, 40, 251-255.

CoLonIUs, H. (1988). Modeling the redundant signals effect by specifying the hazard function. Perception \& Psychophysics, 43, 604-606.

Diederich, A., \& CoLonius, H. (1987). Intersensory facilitation in the motor component? Psychological Research, 49, 23-29.

FELLER, W. (1949). Fluctuation theory of recurrent events. Transactions of the American Mathematical Society, 67, 98-119.

Feller, W. (1966). An introduction to probability theory and its applications (Vol. 2). New York: Wiley.

Gielen, S. C. A. M., Schmidt, R. A., \& VAN den Heuvel, P. J. M. (1983). On the nature of intersensory facilitation of reaction time. Perception \& Psychophysics, 34, 161-168.

GreeN, D. M., LuCE, R. D. (1974). Timing and counting mechanisms in auditory discrimination and reaction time. In D. H. Krantz, R. C. Atkinson, R. D. Luce, \& P. Suppes (Eds.), Contemporary developments in mathematical psychology (Vol. 2, pp. 372-415). San Francisco: Freeman.

Grice, G. R., Canham, L., \& Boroughs, J. M. (1984). Combination rule for redundant information in reaction time tasks with divided attention. Perception \& Psychophysics, 35, 451-463.

LuCE, R. D. (1986). Response times. New York: Oxford University Press.

MilleR, J. O. (1982). Divided attention: Evidence for coactivation with redundant signals. Cognitive Psychology, 14, 247-279.

MilleR, J. [O.] (1986). Timecourse of coactivation in bimodal divided attention. Perception \& Psychophysics, 40, 331-343.

RAAB, D. (1962). Statistical facilitation of simple reaction times. Transactions of the New York Academy of Sciences, 24, 574-590.

RATCLIFF, R. (1988). Continuous versus discrete information processing: Modelling accumulation of partial information. Psychological Review, 95, 238-255.

Smith, W. L. (1988). Renewal theory. In S. Kotz \& N. L. Johnson (Eds.), Encyclopedia of statistical sciences (Vol. 8, pp. 30-36). New York: Wiley.

ULRICH, R., \& GIRAY, M. (1986). Separate-activation models with variable base times: Testability and checking of cross-channel dependency. Perception \& Psychophysics, 39, 248-254.

ULRICH, R., \&TAPF, K. H. (1984). A double-response paradigm to study stimulus intensity effects upon the motor system in simple reaction time experiments. Perception \& Psychophysics, 36, 545-558.

\section{NOTE}

1. After the submission of this note, R. Ulrich and $M$. Giray informed me that they had discussed a special case of the superposition model, namely of exponential intercount times and $\tau=0$, in a speech given in 1983 , and that they are currently planning a separate publication. At the time of submission, I was not aware of this fact. Apparently, one of the reviewers, Jeff Miller, also had explored independently some aspects of the superposition model.

\section{APPENDIX A}

To derive Equation 2, note first that by definition of an additive process, $N_{R}(t)$ is the convolution of $N_{A}(t)$ and $N_{V}(t)$;

$$
P\left[N_{R}(t)=n\right]=\sum_{i=0}^{n} P\left[N_{A}(t)=i\right] \cdot P\left[N_{V}(t)=n-i\right] .
$$

Using an identity originally due to Feller (1949), we have

$$
P_{k}\left(T_{i} \leq t\right)=P\left[N_{k}(t) \geq i\right]=1-\sum_{j=0}^{i-1} P\left[N_{k}(t)=j\right],
$$

and, similarly,

$$
P_{k}\left(T_{i+1} \leq t\right)=P\left[N_{k}(t) \geq(i+1)\right]=1-\sum_{j=0}^{i} P\left[N_{k}(t)=j\right] ;
$$

and so, by subtraction,

$$
P_{k}\left(T_{i} \leq t\right)-P_{k}\left(T_{i+1} \leq t\right)=P\left[N_{k}(t)=i\right] .
$$

To obtain $P_{R}\left(T_{c} \leq t\right)$ in terms of the single stimulus distributions, $P_{A}, P_{V}$, we use Equation a to rewrite Equation b with $k=R$

$$
\begin{aligned}
1-P_{R}\left(T_{c} \leq t\right) & =\sum_{n=0}^{c-1} P\left[N_{R}(t)=n\right] \\
& =\sum_{n=0}^{c-1} \sum_{i=0}^{n} P\left[N_{A}(t)=i\right] \cdot P\left[N_{V}(t)=n-i\right],
\end{aligned}
$$

and we apply Equation $c$ to obtain finally

$$
\begin{aligned}
1-P_{R}\left(T_{c} \leq t\right)= & \sum_{n=0}^{c-1} \sum_{i=0}^{n}\left[P_{A}\left(T_{i} \leq t\right)-P_{A}\left(T_{i+1} \leq t\right)\right] \\
& \cdot\left[P_{V}\left(T_{n-i} \leq t\right)-P_{V}\left(T_{n-i+1} \leq t\right)\right]
\end{aligned}
$$

\section{APPENDIX B}

To derive Equation 4, we again apply Feller's identity to the process $N_{R, \tau}(t): P\left(T_{\mathrm{c}} \leq t\right)=P\left[N_{R, \tau}(t) \geq c\right]$ and use the fact that $N_{R, \tau}(t)$ is Poisson distributed with mean value function $m(t)=$ $\alpha_{V} t$ for $0 \leq t \leq \tau$, and $m(t)=\alpha_{V} \tau+\left(\alpha_{V}+\alpha_{A}\right)(t-\tau)$ for $t \geq \tau$.

To derive $E_{f}\left[T_{c}\right]$, we integrate $1-P_{\tau}\left(T_{c} \leq t\right)$ from zero to infinity (e.g., Feller, 1966, pp. 148, 346) and simplify to obtain Equation 5.

(Manuscript received February 3, 1989; revision accepted for publication April 21, 1989.) 